\title{
Effects of Discrete Time Delays and Parameters Variation on Dynamical Systems
}

\author{
Ibrahim Diakite*, Benito M. Chen-Charpentier ${ }^{\dagger}$ \\ * Department of Global Health and Social Medicine \\ Harvard Medical School, Boston, USA \\ ibrahim_diakite@hms.harvard.edu \\ †Department of Mathematics \\ University of Texas Arlington, Arlington, USA \\ bmchen@uta.edu
}

Received: 29 August 2014, accepted: 20 May 2015, published: 8 June 2015

\begin{abstract}
Delay Differential Equations (DDE's) have received considerable attention in recent years. While most of these articles focused on the effects of the time delays on the stability of the equilibrium points and on the bifurcation that they may raised, very few papers address the key roles that system parameters play on if and how the discrete delays induce stability changes of the equilibria and produce bifurcations near such equilibria. In this article we focus on that question in a general setting, that is, if one has a system of DDE's with one or multiple discrete time delays, what are the results of changing the system parameters values on the effects of the discrete time delays on the dynamic of the system. We present general results for one equation with one and two delays and study a specific example of one equation with one delay. We then establish the procedure for $n$ equations with multiple delays and do a specific example for two equations with two delays. We compute the steady states and analyze their stability as both chosen bifurcation parameters, the discrete time delay $\tau$ and a local equation parameter $\mu$, cross critical values. Our analysis shows that while changes in both parameters can destabilize the steady state, the discrete time delay can only cause stability switches of the steady state for certain values of $\mu$, while
\end{abstract}

the effects of the local equation parameter on the steady state do not necessarily depend on the value of $\tau$. While $\mu$ may cause the system to go through different type of bifurcations, the discrete time delay can only introduce a Hopf bifurcation for certain values of $\mu$.

Keywords-delay differential equations; bifurcation; predator-prey.

\section{INTRODUCTION}

It is well known, that the values of the parameters play a crucial role in the behavior of dynamical systems and that changes in the values can change the behavior significantly. It has also been shown by many researchers (Perelson[1],Allen[2],Bellen[3]) that there is a need to incorporate discrete time delays in dynamical systems (biological systems, physical systems,...) as studied.

Models that incorporate such delays are referred to as delay differential equations (DDE's). DDE's have been extensively studied by many researchers including pioneers Bellman[4], Driver [5], and in more recent years by Culshaw[6], Gakkhar[7], Bellen[3], and a superb monograph on the subject 
by Gopalsamy [8]. While most of these research papers focus on issue of the stability changes caused by the delay(s), the main motivation of this paper is to study how a local bifurcation parameter of the system may affect the changes in stability caused by the delay (s).

Published papers have shown that the incorporation of discrete time delays can highly impact the dynamics of the system, since they can switch the stability of a steady state point, and can also cause the system to go through a Hopf bifurcation near that steady state point (Culshaw[6], Gakkhar[7], Bellen[3]). In this paper we consider a system of $n$ delay differential equations (DDE's) with one parameter $\mu$ as the bifurcation parameter and also with one or more discrete time delays, $\tau$, which can also behave as bifurcation parameters. We are interested in investigating how the parameters $\mu$ and $\tau$ affect the stability of the steady state points of the system, and, more important, how their effects on the system are correlated to each other. We present general results in the one dimensional case (propositions 1 to 3 ) for necessary and sufficient conditions for a stability switch and present a specific example to illustrate these conditions. For the $n$ dimensional case $(n \geq 2)$ we establish the main ideas, but since there are multiple possible cases, we consider only a specific example. We present a non-Kolmogorov type of predator-prey model similar to the model presented by Ruan [9]. In this model we introduce two delays, $\tau_{1}>0$ and $\tau_{2}>0$, to represent the time lag in the growth to maturity of the prey, and the time lag in the growth to maturity of the predator, respectively. We show how the dynamics of the system change depending on certain conditions on $\tau_{1}$ and on another bifurcation parameter $R$. We also point out conditions for the system to go through stability changes when both delays $\tau_{1}$ and $\tau_{2}$ are non-zero. We present necessary conditions for the system to go through a Hopf bifurcation for $\tau_{1}>0$ and $\tau_{2}=0$. Finally we show numerical results illustrating the theoretical results.

\section{OnE Dimensional FiELD}

\section{A. One Equation with One Delay}

Consider the one dimensional delay differential equation with the time delay $\tau$, and the parameter $\mu$ as bifurcation parameters:

$$
\frac{d X}{d t}=f(X(t), X(t-\tau), \mu)
$$

where $f$ is assumed to be smooth enough to guarantee the existence and uniqueness of solutions to (1) under the initial condition (R. Bellman and K. L. Cooke [4])

$$
X(\theta)=\phi(\theta), \quad \theta \in[-\tau, 0] .
$$

Unfortunately equation (1) is too general to analyze. Therefore we will consider a more special form:

$$
\frac{d X}{d t}=f_{1}(X(t), \mu)+f_{2}(X(t-\tau), \mu) .
$$

This form has the advantage that it simplifies the analytical work and also it is the form present in many population dynamical models involving delays [6], [7], [9], [10]. The DDE (2) may or may not have equilibrium points (or steady states) and these will depend on the values of $\mu$. Let $\mu^{*} \in D_{\mu}=\left\{\mu \in R: f\left(X^{*}, X^{*}, \mu\right)=0\right.$ exists $\}$ ,that is $\mu^{*}$ is in the range of values of $\mu$ for which the DDE has an equilibrium point $X^{*}$, i.e., $f\left(X^{*}, X^{*}, \mu^{*}\right)=0$. We are interested in studying the stability of such equilibrium point. In particular, in studying the effect of the parameter $\mu$ and of the discrete time $\tau$ on its stability. To do this we linearize the DDE around the equilibrium point. The characteristic equation is :

$$
\lambda-\left.\frac{d f_{1}}{d X}\right|_{\left(X^{*}, \mu^{*}\right)}-\left.\frac{d f_{2}}{d X}\right|_{\left(X^{*}, \mu^{*}\right)} e^{-\lambda \tau}=0,
$$

and the stability of the equilibrium point $\left(X^{*}, \mu^{*}\right)$ is determined by the sign of the real part of the eigenvalues $\lambda$ of equation (3). 
1) Stability of the Steady State: If $\tau=0$ then the characteristic equation (3) becomes

$$
\lambda-\left.\frac{d f_{1}}{d X}\right|_{\left(X^{*}, \mu^{*}\right)}-\left.\frac{d f_{2}}{d X}\right|_{\left(X^{*}, \mu^{*}\right)=0}
$$

The stability of the steady state then depends only on values of $\mu^{*}$ within $D_{\mu}$. We have two cases:

(a) The steady state $\left(X^{*}, \mu^{*}\right)$ is stable if

$$
\left.\frac{d f_{1}}{d X}\right|_{\left(X^{*}, \mu^{*}\right)}+\left.\frac{d f_{2}}{d X}\right|_{\left(X^{*}, \mu^{*}\right)}<0 \text {. }
$$

(b) The steady state $\left(X^{*}, \mu^{*}\right)$ is unstable if $\left.\frac{d f_{1}}{d X}\right|_{\left(X^{*}, \mu^{*}\right)}+\left.\frac{d f_{2}}{d X}\right|_{\left(X^{*}, \mu^{*}\right)}>0$.

Assume that condition (a) holds, namely the steady state $\left(X^{*}, \mu^{*}\right)$ is stable when there is no delay $(\tau=0)$. We want to know if there exists $\tau>0$ for which the steady state will lose stability. So for $\tau \geq 0$, let $\lambda(\tau)=\alpha(\tau)+i \omega(\tau)$. The characteristic equation (3) becomes:

$$
\begin{aligned}
\alpha+i \omega=\left.\frac{d f_{1}}{d X}\right|_{\left(X^{*}, \mu^{*}\right)}+ & \left.\frac{d f_{2}}{d X}\right|_{\left(X^{*}, \mu^{*}\right)} e^{-\alpha \tau} \cos \omega \tau+ \\
& \left.i \frac{d f_{2}}{d X}\right|_{\left(X^{*}, \mu^{*}\right)} e^{-\alpha \tau} \sin \omega \tau,
\end{aligned}
$$

where, for clarity in the notation, we have not explicitly shown the dependence on $\tau$. Separating the real and imaginary parts, we have:

$$
\begin{aligned}
& \alpha=\left.\frac{d f_{1}}{d X}\right|_{\left(X^{*}, \mu^{*}\right)}+\left.\frac{d f_{2}}{d X}\right|_{\left(X^{*}, \mu^{*}\right)} e^{-\alpha \tau} \cos \omega \tau, \\
& \omega=\left.\frac{d f_{2}}{d X}\right|_{\left(X^{*}, \mu^{*}\right)} e^{-\alpha \tau} \sin \omega \tau .
\end{aligned}
$$

The steady state will lose stability when the real part of the eigenvalue $\lambda$ crosses the zero axis from negative to positive as $\tau$ passes a critical value. By Rouche's Theorem (Dieudonne[11], Theorem 9.17.4) and by the continuity in $\tau$, the transcendental equation (3) has roots with positive real parts if and only if it has pure imaginary roots. Therefore, we look at when the real part of the eigenvalue $\lambda$ becomes zero. In other words, we want to find if there exists a $\tau_{c}>0$ such that $\alpha\left(\tau_{c}\right)=0$. Since

$$
\alpha(0)=\left.\frac{d f_{1}}{d X}\right|_{\left(X^{*}, \mu^{*}\right)}+\left.\frac{d f_{2}}{d X}\right|_{\left(X^{*}, \mu^{*}\right)},
$$

and $\alpha(0)<0$ by assumption (a), therefore if $\tau_{c}>$ 0 exists such that $\alpha\left(\tau_{c}\right)=0$ then by the continuity
(Michael Y. Li and Hogying Shu [10]) of $\alpha$ we have:

- $\alpha(\tau)<0$ for any $0 \leq \tau<\tau_{c}$,

- $\alpha(\tau)>0$ for any $\tau>\tau_{c}$.

Namely the steady state $\left(X^{*}, \mu^{*}\right)$ will lose stability as the delay parameter $\tau$ crosses a critical value $\tau_{c}$. Such $\tau_{c}$ exists if and only if $\alpha\left(\tau_{c}\right)=0$ and $\omega\left(\tau_{c}\right)=\omega_{c}$ satisfies :

$$
\begin{aligned}
\left.\frac{d f_{1}}{d X}\right|_{\left(X^{*}, \mu^{*}\right)} & =-\left.\frac{d f_{2}}{d X}\right|_{\left(X^{*}, \mu^{*}\right)} \cos \omega_{c} \tau_{c} \\
\omega_{c} & =\left.\frac{d f_{2}}{d X}\right|_{\left(X^{*}, \mu^{*}\right)} \sin \omega_{c} \tau_{c} .
\end{aligned}
$$

Squaring equations (7) and (8), and adding them up, we obtain:

$$
\omega_{c}^{2}=\left[\left.\frac{d f_{2}}{d X}\right|_{\left(X^{*}, \mu^{*}\right)}\right]^{2}-\left[\left.\frac{d f_{1}}{d X}\right|_{\left(X^{*}, \mu^{*}\right)}\right]^{2} .
$$

If equation (9) has at least a positive root $\omega_{c}$, then there exists a $\tau_{c}>0$ such that $\alpha(\tau)>0$ whenever $\tau>\tau_{c}$ (see proof in Appendix A). An important question we want to address is, since equation (9) depends on the bifurcation parameter $\mu^{*}$, can one chose $\mu^{*}$ within $D_{\mu}$ so that equation $(9)$ does not have a positive root $\omega_{c}$ ? That is, are there values of $\mu^{*}$ within $D_{\mu}$ such that the delay does not have any effect on the stability of the steady state $\left(X^{*}, \mu^{*}\right)$ ? This question motivates the following propositions (see Appendix B for the proof).

Proposition 1: Consider the one dimensional delay differential equation

$$
\frac{d X}{d t}=f_{1}(X(t), \mu)+f_{2}(X(t-\tau), \mu) .
$$

And assume that the steady state $\left(X^{*}, \mu^{*}\right)$ is stable for $\tau=0$ then we have

(i) If $\left.\frac{d f_{1}}{d X}\right|_{\left(X^{*}, \mu^{*}\right)}<0$ and $\left.\frac{d f_{2}}{d X}\right|_{\left(X^{*}, \mu^{*}\right)}>0$ then the steady state $\left(X^{*}, \mu^{*}\right)$ remains stable for all $\tau \geq 0$.

(ii) If $\left.\frac{d f_{1}}{d X}\right|_{\left(X^{*}, \mu^{*}\right)}>0$ and $\left.\frac{d f_{2}}{d X}\right|_{\left(X^{*}, \mu^{*}\right)}<0$ then there exists a critical value of the delay such that the steady state loses stability as the delay crosses its critical value.

(iii) If $\left.\frac{d f_{1}}{d X}\right|_{\left(X^{*}, \mu^{*}\right)}<0$ and $\left.\frac{d f_{2}}{d X}\right|_{\left(X^{*}, \mu^{*}\right)}<0$ then: 
(a) the steady state remains stable for all $\tau \geq 0$ if

$$
\left.\left|\frac{d f_{2}}{d X}\right|_{\left(X^{*}, \mu^{*}\right)}|<| \frac{d f_{1}}{d X}\right|_{\left(X^{*}, \mu^{*}\right)} \mid,
$$

(b) there exists a $\tau_{c}>0$ such that the steady state becomes unstable for all $\tau>\tau_{c}$ if

$$
\left.\left|\frac{d f_{2}}{d X}\right|_{\left(X^{*}, \mu^{*}\right)}|>| \frac{d f_{1}}{d X}\right|_{\left(X^{*}, \mu^{*}\right)} \mid
$$

Proposition 2: Consider the one dimensional delay differential equation

$$
\frac{d X}{d t}=f_{1}(X(t), \mu)+f_{2}(X(t-\tau), \mu)
$$

And assume that the steady state $\left(X^{*}, \mu^{*}\right)$ is stable for $\tau=0$, that conditions of proposition $1($ iii $)$ hold, and that further more for some $\mu^{*}$ within $D_{\mu}$ we have:

$$
\begin{aligned}
& \left.\frac{d f_{1}}{d X}\right|_{\left(X^{*}, \mu^{*}\right)}=g\left(X^{*}\right) \mathcal{O}\left(\mu^{*}\right), \\
& \left.\frac{d f_{2}}{d X}\right|_{\left(X^{*}, \mu^{*}\right)}=h\left(X^{*}\right) \mathcal{O}\left(\frac{1}{\mu^{*}}\right),
\end{aligned}
$$

then there exists a critical value for $\mu^{*}$ within $D_{\mu}$ such that the steady state $\left(X^{*}, \mu^{*}\right)$ will stay stable for all $\tau \geq 0$ when $\mu^{*}>\mu_{c}$.

2) Example: Consider the one dimensional DDE

$$
\begin{cases}\frac{d Y}{d t}=\mu \frac{Y(t)}{Y(t)+1}-\frac{1}{\mu} Y(t-\tau)^{2}, & \text { if } \quad \mu \neq 0 \\ Y(t)=0, & \text { if } \quad \mu=0\end{cases}
$$

where $\mu$ is a bifurcation parameter and $\tau \geq 0$ is a discrete time delay. For $\mu \in D_{\mu}=R$, the equation has two non-negative equilibrium points: the trivial one $Y_{0}^{*}=0$, and the positive equilibrium point $Y_{1}^{*}=\frac{-1+\sqrt{1+4 \mu^{2}}}{2}$. The characteristic equation is given as

$$
\lambda-\mu \frac{1}{\left(Y^{*}+1\right)^{2}}-\frac{2}{\mu} Y^{*} e^{-\lambda \tau}=0 .
$$

- For the trivial equilibrium point $Y^{*}=0$, its stability only depends on $\mu$ since equation (10) evaluated at $Y^{*}=0$ becomes $\lambda=\mu$. The trivial equilibrium is unstable for $\mu>0$ and all $\tau \geq 0$.
The trivial equilibrium is stable for $\mu<0$ and all $\tau \geq 0$.

- At $Y_{1}^{*}=\frac{-1+\sqrt{1+4 \mu^{2}}}{2}$, equation 10$\}$ becomes:

$$
\lambda-\frac{4 \mu}{\left(1+\sqrt{1+4 \mu^{2}}\right)^{2}}+\frac{\sqrt{1+4 \mu^{2}}-1}{\mu} e^{-\lambda \tau}=0,
$$

then the stability of $Y_{1}^{*}$ depends on both $\mu$ and $\tau$.

1) If $\tau=0$ then equation (11) becomes

$$
\lambda=-\frac{4 \mu \sqrt{1+4 \mu^{2}}}{\left(1+\sqrt{1+4 \mu^{2}}\right)^{2}}
$$

then

$\lambda<0$ if $\mu>0$, therefore the equilibrium $Y_{1}^{*}$ is stable (Fig 2)

$\lambda>0$ if $\mu<0$, therefore the equilibrium $Y_{1}^{*}$ is unstable (Fig 2).

Remark: To better understand the situation, the stability of both equilibria when there is no delay is shown in the following table:

TABLE I

STABILITY REGIONS

\begin{tabular}{ccc}
\hline \hline Case & $Y_{0}^{*}=0$ & $Y_{1}^{*}=\frac{-1+\sqrt{1+4 \mu^{2}}}{2}$ \\
\hline$\mu<0$ & stable & unstable \\
$\mu=0$ & stable & stable \\
$\mu>0$ & unstable & stable \\
\hline
\end{tabular}

At the equilibrium $(Y, \mu)=(0,0)$, there is an exchange of stability. This is a transcritical bifurcation (Guckenheimer[12]). Geometrically, there are two curves of equilibria which intersect at the origin and lie on both sides of $\mu=0$. Stability of the equilibrium changes along either curve on passing through $\mu=0$.

2) If $\tau>0$ and $\lambda(\tau)=\alpha(\tau)+i \omega(\tau)$, there exists a critical $\tau_{c}$ such that $\alpha\left(\tau_{c}\right)=0$ and $\lambda\left(\tau_{c}\right)= \pm i \omega\left(\tau_{c}\right)= \pm i \omega_{c}$ (a pair of pure 


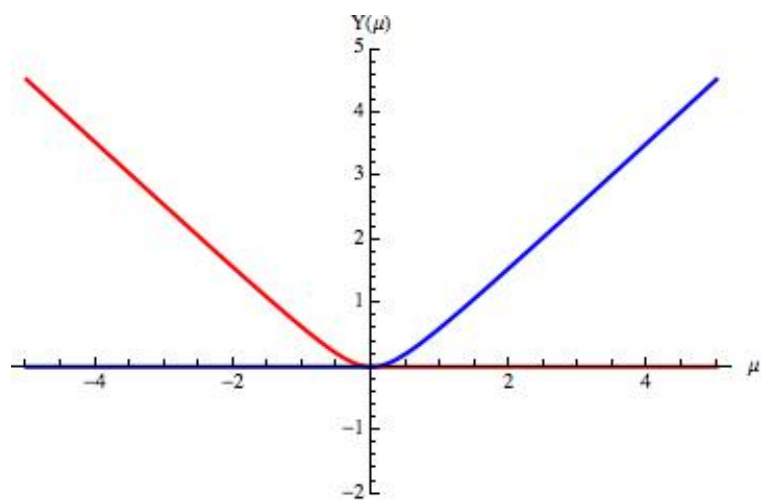

Fig. 1. Transcritical bifurcation around $\mu=0$. Unstable equilibrium (red), and Stable equilibrium (blue).

imaginary eigenvalues) is solution of (8) if and only if

$\omega_{c}^{2}=\frac{16\left[\mu^{2}\left(1+\sqrt{1+4 \mu^{2}}\right)^{2}-1\right]}{\left(1+\sqrt{1+4 \mu^{2}}\right)^{4}}$ has a positive root $\omega_{c}$,

and that is the case if and only if $\mu^{2}(1+$ $\left.\sqrt{1+4 \mu^{2}}\right)^{2}-1>0$.

(2a) If $\mu \geq \frac{1}{2}$ then $\mu^{2}\left(1+\sqrt{1+4 \mu^{2}}\right)^{2}-1>0$ therefore there exists $\tau_{c}>0$ such that the equilibrium loses stability whenever $\tau>\tau_{c}$ (Fig 3 left).

(2b) If $\mu \leq-\frac{1}{2}$ then $\mu^{2}\left(1+\sqrt{1+4 \mu^{2}}\right)^{2}-1>$ 0 therefore there exists $\tau_{c}>0$ such that the equilibrium gains stability whenever $\tau>\tau_{c}$.

(2c) If $-\frac{1}{2}<\mu<\frac{1}{2}$ then $\mu^{2}(1+$ $\left.\sqrt{1+4 \mu^{2}}\right)^{2}-1<0$ therefore the delay has no effect on the stability of the equilibrium.

For $\mu \geq \frac{1}{2}$, the equilibrium is unstable for all $\tau>$ 0.55 , and for $0<\mu<\frac{1}{2}$ the equilibrium remains stable for all $\tau$.

\section{B. One Equation with Multiple Delays}

Consider the one dimensional delay differential equation with the time lags $\tau_{k}, \quad k=1,2, \ldots$, and $\mu$ as bifurcation parameters:

$\frac{d X}{d t}=f_{1}(X(t), \mu)+f_{2}\left(X\left(t-\tau_{1}\right), \ldots, X\left(t-\tau_{k}\right), \mu\right)$
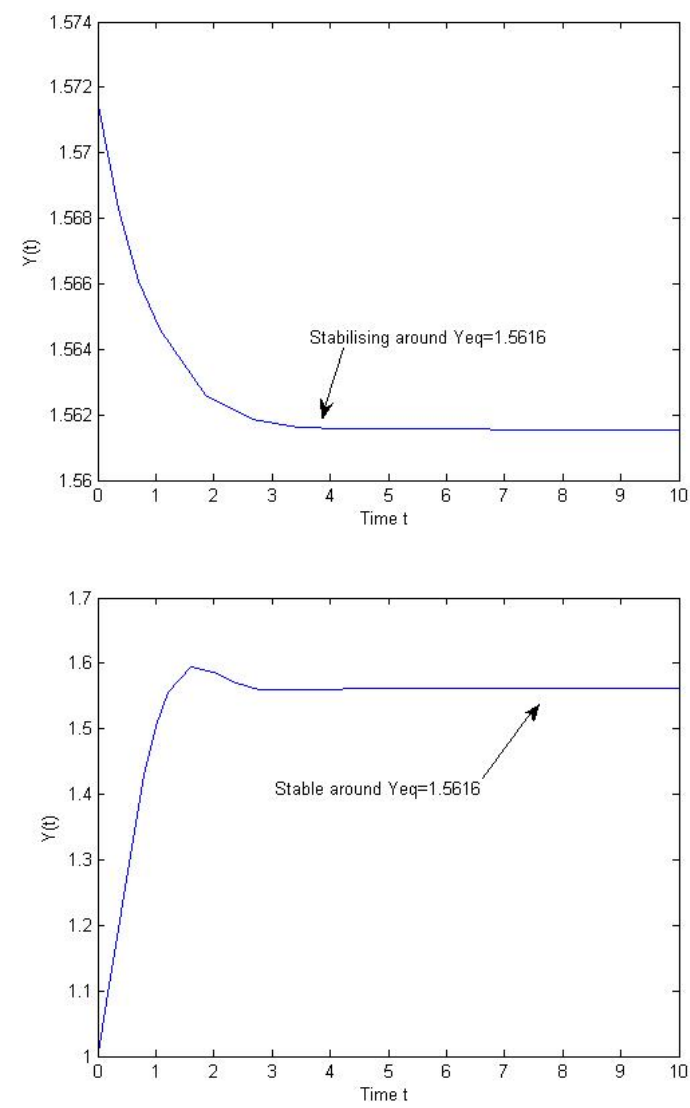

Fig. 2. The positive equilibrium is stable for $\tau=0$ and $\mu=2$, top graph. The equilibrium still remains stable for $\tau=0.4\left(\tau<\tau_{c}=0.55\right)$ and $\mu=2$, bottom graph

Let $\left(X^{*}, \mu^{*}\right)=\left(X^{*}, X^{*}, \ldots, X^{*}, \mu^{*}\right)$ be the steady state of equation 12 , i.e., $f_{1}\left(X^{*}, \mu^{*}\right)+$ $f_{2}\left(X^{*}, X^{*}, \ldots, X^{*}, \mu^{*}\right)=0$. To study the stability of the steady state we compute the characteristic equation:

$$
\lambda-\left.\frac{d f_{1}}{d X}\right|_{\left(X^{*}, \mu^{*}\right)}-\left.\sum_{j=1}^{k} \frac{d f_{2}}{d X}\right|_{\left(X^{*}, \mu^{*}\right)} e^{-\lambda \tau_{j}}=0 .
$$

For clarity of the presentation we consider the case of only two delays. Therefore the characteristic equation is written as

$\lambda-\left.\frac{d f_{1}}{d X}\right|_{\left(X^{*}, \mu^{*}\right)}-\left.\frac{d f_{2}}{d X}\right|_{\left(X^{*}, \mu^{*}\right)}\left(e^{-\lambda \tau_{1}}+e^{-\lambda \tau_{2}}\right)=0$ 
Note that if $\tau_{1}=\tau_{2}=\tau$ or $\tau_{1}=0$ or $\tau_{2}=$ 0 then we are back to the previous case of one equation with one delay. We will assume that $\tau_{1}$ is in its stable domain, i.e., $0<\tau_{1}<\tau_{1 c}$ and $\tau_{2}>0$. We now examine how variation of $\tau_{2}$ and $\mu$ affects the stability of the steady state. Consider $\lambda\left(\tau_{2}\right)=\alpha\left(\tau_{2}\right)+i \omega\left(\tau_{2}\right)$ as solution of equation (14). We look for a critical value $\tau_{2 c}$ of $\tau_{2}$ such that $\alpha\left(\tau_{2 c}\right)=0$ and $\lambda\left(\tau_{2 c}\right)=i \omega\left(\tau_{2 c}\right)=i \omega_{2 c}$ is solution of equation (14). Such $\tau_{2 c}$ exists if and only if:

$$
\begin{array}{r}
i \omega_{2 c}-\left.\frac{d f_{2}}{d X}\right|_{\left(X^{*}, \mu^{*}\right)}\left(\cos \omega_{2 c} \tau_{1}-i \sin \omega_{2 c} \tau_{1}\right) \\
-\left.\frac{d f_{2}}{d X}\right|_{\left(X^{*}, \mu^{*}\right)}\left(\cos \omega_{2 c} \tau_{2 c}-i \sin \omega_{2 c} \tau_{2 c}\right) \\
-\left.\frac{d f_{1}}{d X}\right|_{\left(X^{*}, \mu^{*}\right)}
\end{array}
$$

Separate real and imaginary parts:

$$
\begin{gathered}
-\left.\frac{d f_{2}}{d X}\right|_{\left(X^{*}, \mu^{*}\right)} \\
\cos \omega_{2 c} \tau_{2 c}= \\
\left.\frac{d f_{2}}{d X}\right|_{\left(X^{*}, \mu^{*}\right)} \cos \omega_{2 c} \tau_{1},+\left.\frac{d f_{1}}{d X}\right|_{\left(X^{*}, \mu^{*}\right)} \\
\left.\frac{d f_{2}}{d X}\right|_{\left(X^{*}, \mu^{*}\right)} \sin \omega_{2 c} \tau_{2 c}= \\
-\left.\frac{d f_{2}}{d X}\right|_{\left(X^{*}, \mu^{*}\right)} \sin \omega_{2 c} \tau_{1}-\omega_{2 c} .
\end{gathered}
$$

Adding the square of (16) and (II-B) we have

$$
\begin{array}{r}
{\left[\left.\frac{d f_{2}}{d X}\right|_{\left(X^{*}, \mu^{*}\right)}\right]^{2}=\left(\omega_{2 c}+\left.\frac{d f_{2}}{d X}\right|_{\left(X^{*}, \mu^{*}\right)} \sin \omega_{2 c} \tau_{1}\right)^{2}} \\
+\left(\left.\frac{d f_{1}}{d X}\right|_{\left(X^{*}, \mu^{*}\right)}+\left.\frac{d f_{2}}{d X}\right|_{\left(X^{*}, \mu^{*}\right)} \sin \omega_{2 c} \tau_{1}\right)^{2}
\end{array}
$$

Clearly $\tau_{2 c}$ exists if and only the function:

$$
\begin{aligned}
H\left(\omega_{2 c}\right) & =\left(\omega_{2 c}+\left.\frac{d f_{2}}{d X}\right|_{\left(X^{*}, \mu^{*}\right)} \sin \omega_{2 c} \tau_{1}\right)^{2} \\
& +\left(\left.\frac{d f_{1}}{d X}\right|_{\left(X^{*}, \mu^{*}\right)}+\left.\frac{d f_{2}}{d X}\right|_{\left(X^{*}, \mu^{*}\right)} \cos \omega_{2 c} \tau_{1}\right)^{2} \\
& -\left[\left.\frac{d f_{2}}{d X}\right|_{\left(X^{*}, \mu^{*}\right)}\right]^{2}
\end{aligned}
$$

has at least a positive root.
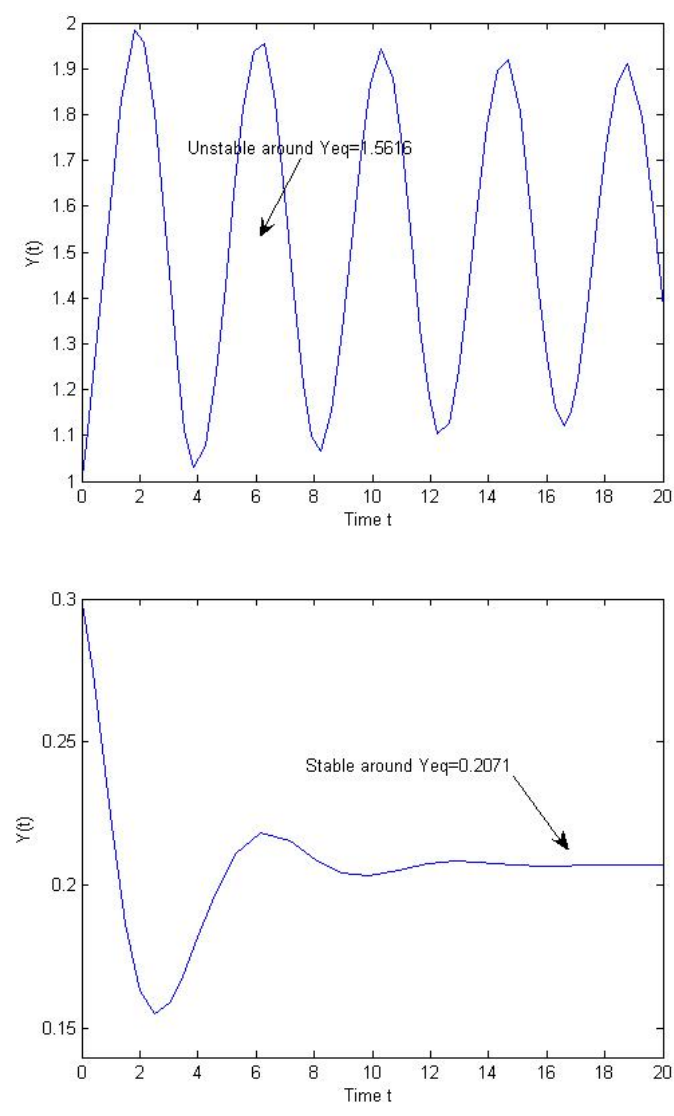

Fig. 3. For $\mu=2$ and $\tau=0.9$ the equilibrium is unstable, top graph. For $\mu=0.2$ and $\tau=1$ the equilibrium is stable, bottom graph

Proposition 3: Consider the one dimensional delay differential equation with the time lag $\tau_{1}$, $\tau_{2}$, and $\mu$ as bifurcation parameters:

$\frac{d X}{d t}=f_{1}(X(t), \mu)+f_{2}\left(X\left(t-\tau_{1}\right), X\left(t-\tau_{2}\right), \mu\right)$.

Assume that the steady state $\left(X^{*}, \mu^{*}\right)=$ $\left(X^{*}, X^{*}, \mu^{*}\right)$ of 19$]$ is stable for $0<\tau_{1}<\tau_{1 c}$. If $\left.\frac{d f_{2}}{d X}\right|_{\left(X^{*}, \mu^{*}\right)}>0$ and $\left.\frac{d f_{1}}{d X}\right|_{\left(X^{*}, \mu^{*}\right)}<0$, then there exists a critical value $\tau_{2 c}>0$ for $\tau_{2}$ such that $\left(X^{*}, \mu^{*}\right)$ losses stability as $\tau_{2}$ crosses $\tau_{2 c}$.

Proof: Such $\tau_{2 c}$ exists if and only if equation $H\left(\omega_{2 c}\right)=0$ has at least a positive equation. Or 
If $\left.\frac{d f_{2}}{d X}\right|_{\left(X^{*}, \mu^{*}\right)}>0$ and $\left.\frac{d f_{1}}{d X}\right|_{\left(X^{*}, \mu^{*}\right)}<0$ then

$$
\begin{aligned}
H(0)=\left(\left.\frac{d f_{1}}{d X}\right|_{\left(X^{*}, \mu^{*}\right)}\right. & \left.+\left.\frac{d f_{2}}{d X}\right|_{\left(X^{*}, \mu^{*}\right)}\right)^{2} \\
& -\left(\left.\frac{d f_{2}}{d X}\right|_{\left(X^{*}, \mu^{*}\right)}\right)^{2}<0
\end{aligned}
$$

And also $H\left(\omega_{2 c}\right) \rightarrow \infty$ as $\omega_{2 c} \rightarrow \infty$. Then the intermediate value theorem assures that equation $H\left(\omega_{2 c}\right)=0$ has at least a positive root.

We now extend our analysis to a system of ndelay differential equations with multiple discrete time delays $\tau_{1}, \tau_{2}, \ldots, \tau_{k}$, and a local bifurcation parameter $\mu$.

\section{N DIMENSIONAL FIELD}

Consider the following system non-linear delay differential equations:

$$
\frac{d x}{d t}=f\left(x(t), x\left(t-\tau_{1}\right), \ldots, x\left(t-\tau_{k}\right), \mu\right),
$$

where $x \in \mathbb{R}^{n}, \tau_{j} \geq 0,1 \leq j \leq k$ are constant discrete times,

$f: \mathbb{R}^{n+1} \times C^{k} \rightarrow \mathbb{R}^{n}$ is assumed to be smooth enough to guarantee existence and uniqueness of solutions to 21 under the initial value condition (R. Bellman and K. L. Cooke [4] and J. K. Hale and S. M. Verduyn Lunel [13])

$$
x(\theta)=\phi(\theta), \quad \theta \in[-\tau, 0],
$$

where $C=C\left([-\tau, 0], \mathbb{R}^{n}\right), \tau=\max _{1 \leq j \leq k} \tau_{j}$.

Suppose $f\left(x^{*}, x^{*}, \ldots, x^{*}, \mu^{*}\right)=0$, that is, $\left(x^{*}, \mu^{*}\right)$ is a steady state of system (21). We are interested in studying the stability of such equilibrium point. In particular studying the effect of the parameter $\mu$ and the discrete time delays $\tau_{1}, \tau_{2}, \ldots, \tau_{k}$ on its stability. The linearization of 21) at $\left(x^{*}, \mu^{*}\right)$ has the form (Ruan [9]):

$$
\frac{d X}{d t}=A_{0}\left(\mu^{*}\right) X(t)+\sum_{j=1}^{k} A_{j}\left(\mu^{*}\right) X\left(t-\tau_{j}\right)
$$

where $X \in \mathbb{R}^{n}$, each $A_{j}\left(\mu^{*}\right) \quad(0 \leq j \leq k)$ is an $n \times n$ constant matrix that depends on values of $\mu^{*}$ within $D_{\mu}$. The transcendental equation associated with system 21) is given as :

$$
\operatorname{det}\left[\lambda I-A_{0}\left(\mu^{*}\right)-\sum_{j=1}^{k} A_{j}\left(\mu^{*}\right) e^{-\lambda \tau_{j}}\right]=0
$$

Equation (23) has been studied by many researchers

(Ruan [9], R. Bellman and K. L. Cooke [4] and J. K. Hale and S. M. Verduyn Lunel [13]). The following result, which was proved by Chin [14] for $k=1$ and by Datko [15] and Hale et al. [13] for $k \geq 1$, gives a necessary and sufficient condition for the absolute stability of system (22).

Lemma 1: System (22) is stable for all delays $\tau_{j}(1 \leq j \leq k)$ if and only if

(i) $\operatorname{Re} \lambda\left(\sum_{j=0}^{k} A_{j}\left(\mu^{*}\right)\right)<0$;

(ii) $\operatorname{det}\left[i \omega I-A_{0}\left(\mu^{*}\right)-\sum_{j=1}^{k} A_{j}\left(\mu^{*}\right) e^{-i \omega \tau_{j}}\right] \neq 0$ for all $\omega>0$

Clearly, the stability of the steady state $\left(x^{*}, \mu^{*}\right)$ and the effects of the discrete times $\tau_{j}$ on its stability depend on values of $\mu^{*}$ within $D_{\mu}$. To further investigate the effects of $\mu$, and the discrete time delays $\tau_{j}$ on the stability of $\left(x^{*}, \mu^{*}\right)$, the exact entries of the matrices $A_{j}\left(\mu^{*}\right)$ are needed to avoid doing a large number of cases. Note that the difficulty of the analysis is not due to the number of delays but to the number of equations. Even in the case of two equations with one delay, one needs to consider:

$$
\operatorname{det}\left[\lambda I-A_{0}\left(\mu^{*}\right)-A_{1}\left(\mu^{*}\right) e^{\lambda \tau}=0\right],
$$

where

$$
A_{i}\left(\mu^{*}\right)=\left.\frac{\partial f}{\partial X_{i}}\right|_{\left(X^{*}, \mu^{*}\right)}, \quad i=0,1 .
$$

So the stability depends on all the entries of the $A_{i}, \quad i=0,1$, we have many different cases.

Therefore to present the ideas we consider a specific example with $n=2, k=2$, that is a two dimensional delay differential equations with two discrete time delays, and a local bifurcation parameter. 


\section{A. Two Dimensional Field Example}

Consider the non-Kolmogorov type (Holling) predator-prey model

$$
\begin{aligned}
& \frac{d x}{d t}=r_{1} x\left(t-\tau_{1}\right)-a_{1} \frac{x(t) y(t)}{x(t)+1}, \\
& \frac{d y}{d t}=-r_{2} y(t)+a_{2} \frac{x\left(t-\tau_{2}\right) y\left(t-\tau_{2}\right)}{x\left(t-\tau_{2}\right)+1}
\end{aligned}
$$

where the parameters are described in the following table:

\section{TABLE II}

\section{PARAMETER VALUes}

\begin{tabular}{clc}
\hline \hline Parameters & Description & Values \\
\hline$x(t)$ & the prey population & \\
$y(t)$ & the predator population & \\
$r_{1}$ & the growth rate of the prey in the absence of predators & 0.5 \\
$r_{2}$ & the death rate of predators in the absence of the prey & 0.5 \\
$a_{1}$ & the predation rate of the prey by the predators & 0.5 \\
$a_{2}$ & the conversion rate for the predators & 5 \\
$\tau_{1}$ & the time lag in the growth to maturity of the prey & varies \\
$\tau_{2}$ & the time lag in the growth to maturity of the predators & varies \\
\hline
\end{tabular}

Note that $r_{1}>0, r_{2}>0, a_{1}>0, a_{2}>0, \tau_{1}>0$, $\tau_{2}>0$.

Proposition 4: If the basic reproductive ratio (Ameh[16]) $R>1$, the system has two nonnegative steady states:

$$
\left(x_{0}^{*}, y_{0}^{*}\right)=(0,0) \text {, and }\left(x_{1}^{*}, y_{1}^{*}\right)=\left(\frac{1}{R-1}, \frac{R R^{\prime}}{R-1}\right) \text {, }
$$$$
\text { where } R=\frac{a_{2}}{r_{2}} \quad R^{\prime}=\frac{a_{1}}{r_{1}} \text {. }
$$

We consider $R, \tau_{1}$ and $\tau_{2}$ as the bifurcation parameters for the system 24,25) since changes of them may affect the existence and stability of the equilibrium points.

\section{B. Stability Analysis}

Proposition 5: There exists a critical value for $\tau_{1}$ such that

(i) The steady state $\left(x_{0}^{*}, y_{0}^{*}\right)$ is unstable for $\tau_{1}=$ 0 , and all $\tau_{2} \geq 0$.

(ii) The steady state $\left(x_{0}^{*}, y_{0}^{*}\right)$ is stable for $\tau_{1} \geq$ $\tau_{1 c}$, and all $\tau_{2} \geq 0$.

Proposition 6: If $\left[(b-d)^{2}-r_{1}^{2}-2 a_{1} f\right]<0$ and $\Delta=\left[(b-d)^{2}-r_{1}^{2}-2 a_{1} f\right]^{2}-4 a_{1}^{2} f^{2} \geq 0$ then there exists a critical $\tau_{1 c}^{\prime}$ such that (i) The steady state $\left(x_{1}^{*}, y_{1}^{*}\right)=\left(\frac{1}{R-1}, \frac{R R^{\prime}}{R-1}\right)$ is unstable for $0 \leq \tau_{1}<\tau_{1 c}^{\prime}$ and $\tau_{2}=0$.

(ii) The steady state $\left(x_{1}^{*}, y_{1}^{*}\right)$ is stable for $\tau_{1}>\tau_{1 c}^{\prime}$ and $\tau_{2}=0$.

Note that $\tau_{1}$ affects the stability of the positive equilibrium only for values of $R$ such that conditions $C(0)$ are satisfied.

Remark: For our parameter values, we have

$$
\left[\left(b-r_{2}\right)^{2}-r_{1}^{2}-2 a_{1} f\right]=-0.9475<0 \quad \text { and }
$$

$\Delta=\left[\left(b-r_{2}\right)^{2}-r_{1}^{2}-2 a_{1} f\right]^{2}-4 a_{1}^{2} f^{2}=0.0878>0$

Proposition 7: Consider system 24,25) with $\tau_{1}$ in its unstable interval $\left(0 \leq \tau_{1}<\tau_{1 c}^{\prime}\right)$. If $a_{1} \geq 2$, then there exists a critical $\tau_{2}>0$, such that the positive equilibrium becomes stable for $\tau_{2}>\tau_{2 c}$. Note that the effect of $\tau_{2}$ on the stability of the positive equilibrium does not depend on the values of $R$.

\section{Hopf Bifurcation Analysis}

According to the Hopf Bifurcation Theorem (Culshaw [6]), the discrete time delay $\tau_{1}$ will cause the system to go through a Hopf bifurcation near the steady state $\left(x_{1}^{*}, y_{1}^{*}\right)$, if the following transversality condition is satisfied:

$$
\left.\frac{d \alpha\left(\tau_{1}\right)}{d \tau_{1}}\right|_{\tau_{1}=\tau_{1 c}^{\prime}} \neq 0 .
$$

To check this condition we recall that the characteristic equation of the system at $\left(x_{1}^{*}, y_{1}^{*}\right)$ when $\tau_{2}=0$ is given as :

$$
\lambda^{2}+\left(b-r_{2}\right) \lambda-r_{1} e^{-\lambda \tau_{1}} \lambda+a_{1} f=0 .
$$

Substituting $\lambda\left(\tau_{1}\right)=\alpha\left(\tau_{1}\right)+i \omega\left(\tau_{1}\right)$ in equation (27), we have :

$$
\begin{gathered}
\alpha^{2}-\omega^{2}+2 \alpha \omega i+\left(b-r_{2}\right) \alpha+\left(b-r_{2}\right) \omega i \\
-r_{1} e^{\alpha \tau_{1}}\left(\cos \omega \tau_{1}-i \sin \omega \tau_{1}\right)(\alpha+i \omega)+a_{1} f=0 .
\end{gathered}
$$


I. Diakite et al., Effects of Discrete Time Delays and Parameters ...

We equate the real and the imaginary parts to zero, and we have :

$$
\begin{array}{r}
\alpha^{2}-\omega^{2}+a_{1} f+r_{1} e^{\alpha \tau_{1}}\left(\alpha \cos \omega \tau_{1}+\omega \sin \omega \tau_{1}\right) \\
+\left(b-r_{2}\right) \alpha=0,
\end{array}
$$

$2 \alpha \omega-r_{1} e^{\alpha \tau_{1}}\left(\omega \cos \omega \tau_{1}-\alpha \sin \omega \tau_{1}\right)$

$$
+\left(b-r_{2}\right) \omega=0 \text {. }
$$

We differentiate equations (29) and (30) with respect to $\tau_{1}$ and evaluate at $\tau_{1}=\tau_{1 c}^{\prime}$ for which $\alpha\left(\tau_{1 c}^{\prime}\right)=0$ and $\omega\left(\tau_{1 c}^{\prime}\right)=\omega_{c}^{\prime}$. We obtain

$$
\begin{gathered}
\left.A \frac{d \omega\left(\tau_{1}\right)}{d \tau_{1}}\right|_{\tau_{1}=\tau_{1 c}^{\prime}}-\left.B \frac{d \alpha\left(\tau_{1}\right)}{d \tau_{1}}\right|_{\tau_{1}=\tau_{1 c}^{\prime}}= \\
C \cos \omega_{c}^{\prime} \tau_{1 c}^{\prime}+D \sin \omega_{c}^{\prime} \tau_{1 c}^{\prime} \\
\left.B \frac{d \omega\left(\tau_{1}\right)}{d \tau_{1}}\right|_{\tau_{1}=\tau_{1 c}^{\prime}}+\left.A \frac{d \alpha\left(\tau_{1}\right)}{d \tau_{1}}\right|_{\tau_{1}=\tau_{1 c}^{\prime}}= \\
C \sin \omega_{c}^{\prime} \tau_{1 c}^{\prime}-D \cos \omega_{c}^{\prime} \tau_{1 c}^{\prime}
\end{gathered}
$$

where

$$
\begin{aligned}
& A:=2 \omega_{c}^{\prime}-r_{1} \tau_{1 c}^{\prime} \sin \omega_{c}^{\prime} \tau_{1 c}^{\prime}, \\
& B:=\left(b-r_{2}\right)+r_{1} \tau_{1 c}^{\prime} \cos \omega_{c}^{\prime} \tau_{1 c}^{\prime} \\
& C:=r_{1} \tau_{1 c}^{\prime}\left(\omega_{c}^{\prime 2}+\omega_{c}^{\prime} \tau_{1 c}^{\prime}\right), \\
& D:=r_{1} \tau_{1 c}^{\prime} \omega_{c}^{\prime} \sin \omega_{c}^{\prime} \tau_{1 c}^{\prime} .
\end{aligned}
$$

By solving equations (31) and 32 we have:

$$
\begin{aligned}
& \left.\frac{d \alpha\left(\tau_{1}\right)}{d \tau_{1}}\right|_{\tau_{1}=\tau_{1 c}^{\prime}}= \\
& \frac{(A C-B D) \sin \omega_{c}^{\prime} \tau_{1 c}^{\prime}-(A D+B C) \cos \omega_{c}^{\prime} \tau_{1 c}^{\prime}}{A^{2}+B^{2}} .
\end{aligned}
$$

The system undergoes through a Hopf bifurcation near $\left(x_{1}^{*}, y_{1}^{*}\right)$ if:

$(A C-B D) \sin \omega_{c}^{\prime} \tau_{1 c}^{\prime}-(A D+B C) \cos \omega_{c}^{\prime} \tau_{1 c}^{\prime} \neq 0$.

\section{Numerical Results}

To illustrate the effect of the parameter $R$ and the discrete time delay on the stability of the steady state $\left(x^{*}, y^{*}\right)$, and to support the theoretical predictions discussed above, we conducted numerical simulations for the system 24,25). We used DDE-BIFTOOL (Engelborghs[17]) for the stability and bifurcation analysis and also used the Matlab solvers ode23 and dde23 (Shampine[18],Shampine[19]) to see the behavior of the predator and prey populations through time. All the parameter values are given in Table II.

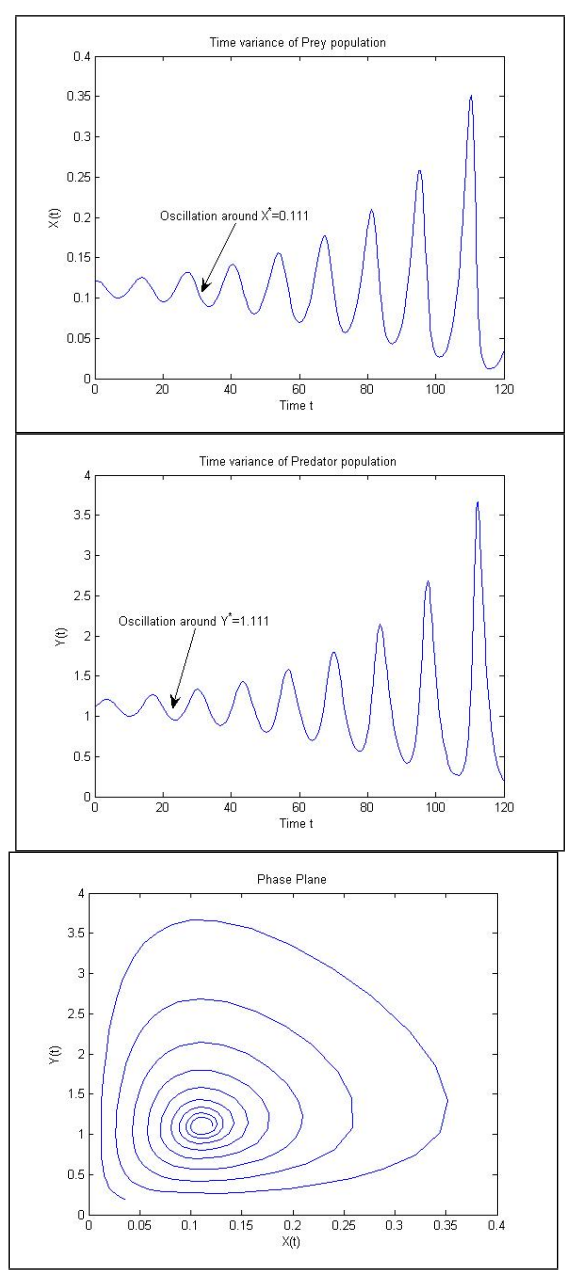

Fig. 4. The positive equilibrium is unstable for $\tau_{1}=\tau_{2}=0$ and $R=10>1$. The system exhibits a spiral out from the equilibrium $\left(x_{1}^{*}, y_{1}^{*}\right)=(0.111,1.111)$. 
For the given parameters values we have $R=$ $10>1$, and a positive equilibrium exists and is given as $\left(x_{1}^{*}, y_{1}^{*}\right)=(0.111,1.111)$. When there is no delay the prey and predator populations variation through time is shown on Figure 4.

For our parameter values we have:

$$
\left[(b-d)^{2}-r_{1}^{2}-4 f\right]=-0.9475<0 \quad \text { and }
$$

$\Delta=\left[(b-d)^{2}-r_{1}^{2}-4 f\right]^{2}-16 f^{2}=0.0878>0$.

Then there exists a $\tau_{1 c}=6$ such that the steady state remains unstable for $0 \leq \tau_{1}<\tau_{1 c}$ and $\tau_{2}=0$ (see Figure 5), it becomes stable as $\tau_{1}$ crosses $\tau_{1 c}$ and $\tau_{2}=0$ as shown on Figure 6 .
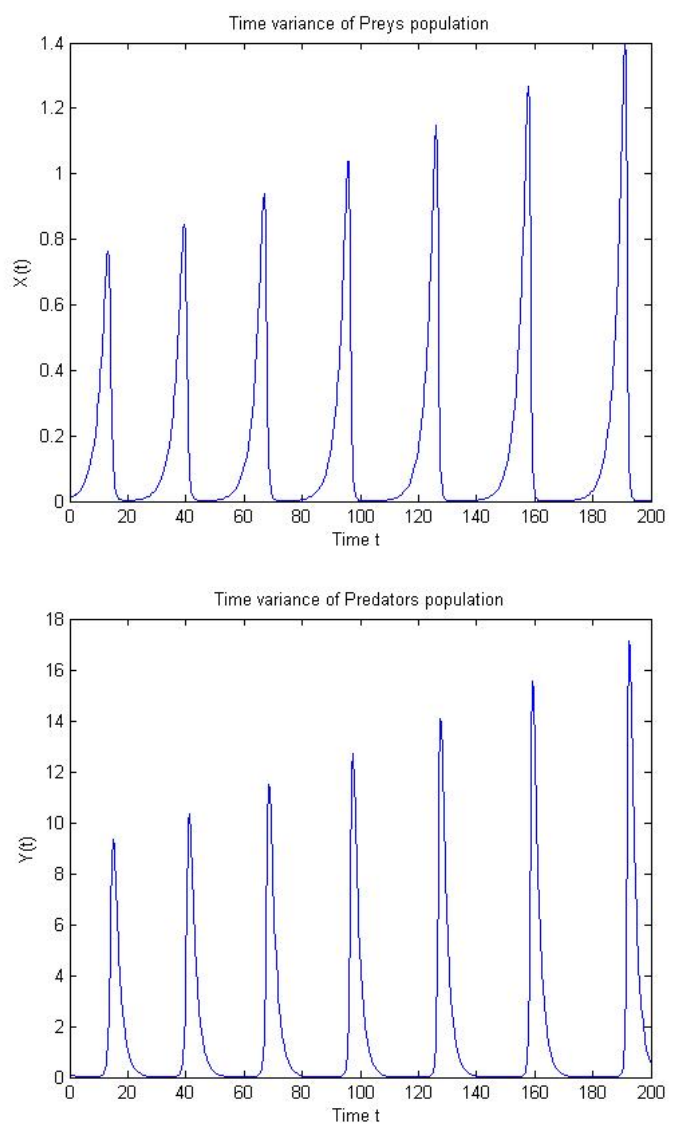

Fig. 5. The positive equilibrium remains unstable for $\tau_{1}=$ $1<\tau_{1 c}=6$ and $\tau_{2}=0$.
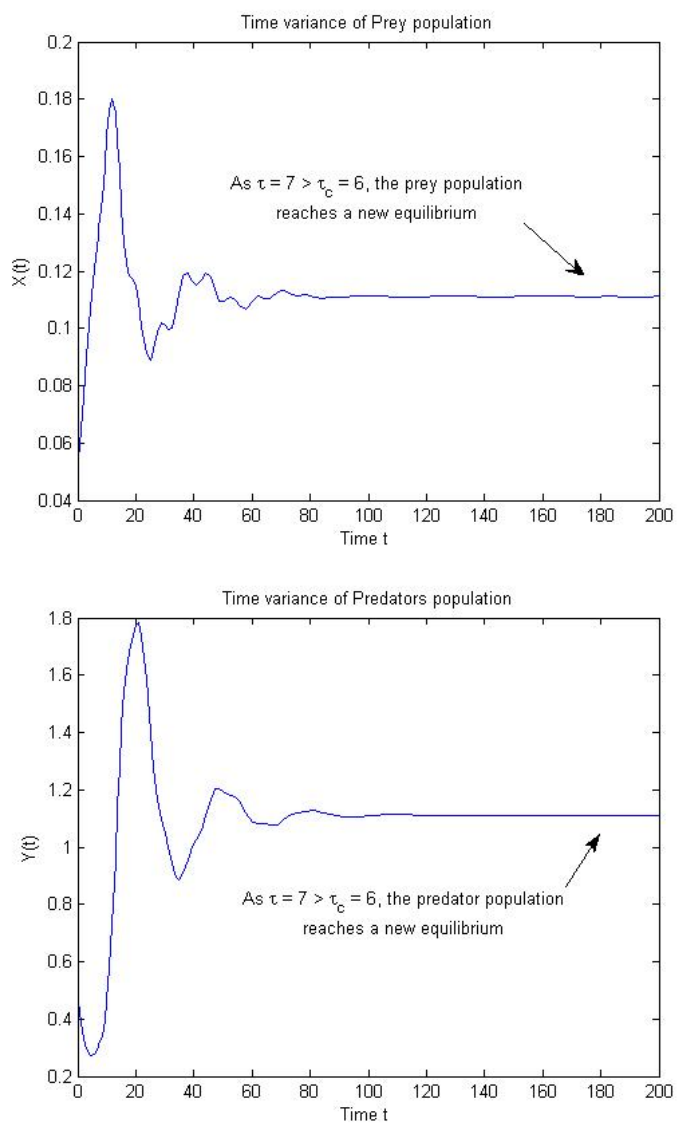

Fig. 6. The positive equilibrium is stable for $\tau_{1}=7>$ $\tau_{1 c}=6$ and $\tau_{2}=0$.

We examine closely the stability switch introduces by $\tau_{1}$. We use DDE-BIFTOOL to compute the eigenvalues of the characteristic equation (38) for $\tau_{2}=0$ and $0 \leq \tau_{1} \leq 10$. In Figure 7] we plot the real parts versus the imaginary parts of these eigenvalues.

We see that the equilibrium $\left(x_{1}^{*}, y_{1}^{*}\right)$ stabilizes as $\tau_{1}$ crosses the critical value $\tau_{1 c}^{\prime}=6$. We also plot in Figure 7 the eigenvalues of equation (38) for $\tau_{1}=\tau_{1 c}^{\prime}=6$ and observe a pair of two pure imaginary eigenvalues. The system undergoes through a Hopf bifurcation as $\tau_{1}$ crosses $\tau_{1 c}^{\prime}$. We compute the Hopf bifurcations branches using Matlab and show them in Figure 8 . 


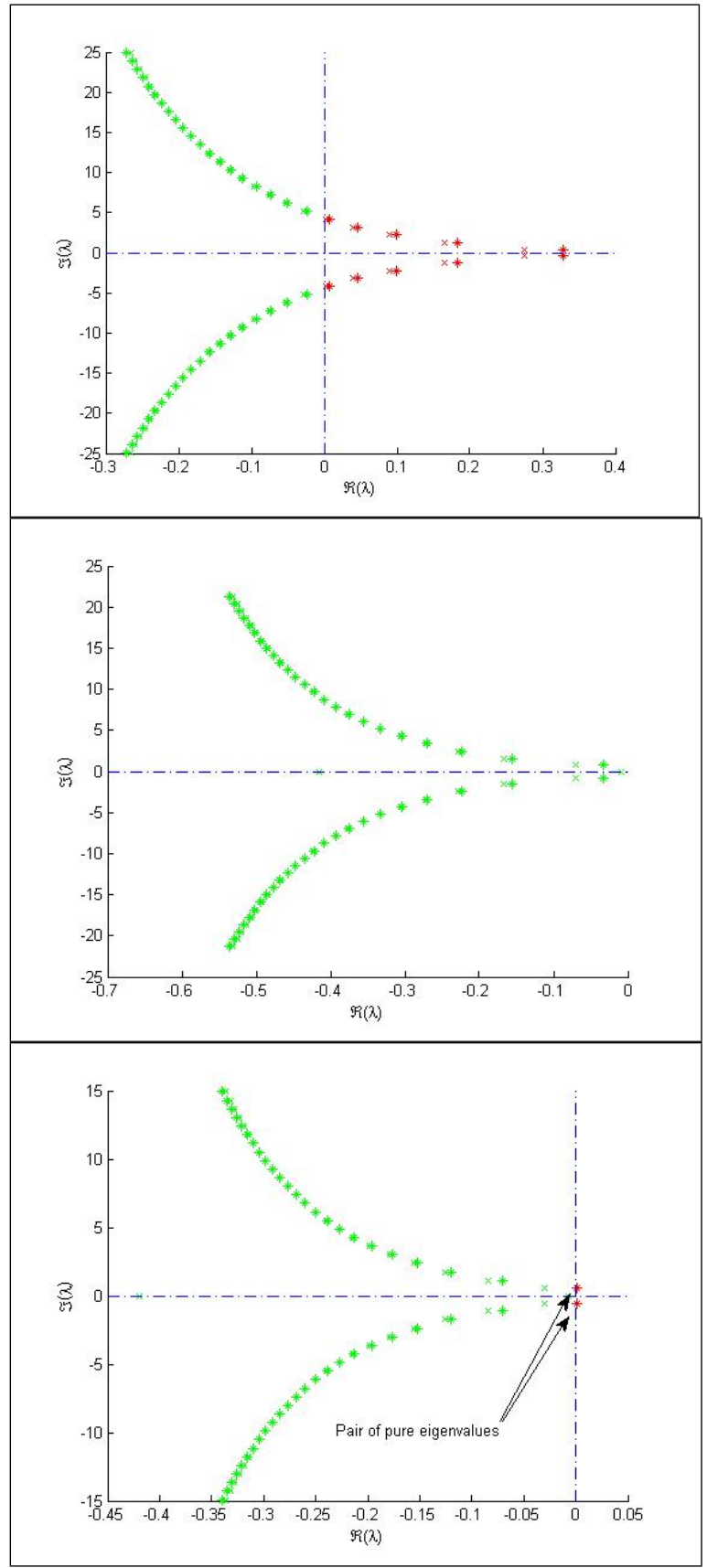

Fig. 7. The eigenvalues of the characteristic equation (38) for $\tau_{1}=3$ (left) and $\tau_{1}=8$ (center) with $\tau_{2}=0$.At $\tau_{1}=\tau_{1 c}^{\prime}=6$ we can clearly observe a pair of 2 pure imaginary eigenvalues (right).

Note $\tau_{1 c}^{\prime}=6$ and $\tau_{2 c}=2.5$ For $\tau_{1}=2$ and $\tau_{2}=$ 0.5 the equilibrium is unstable as shown in Figure 9.

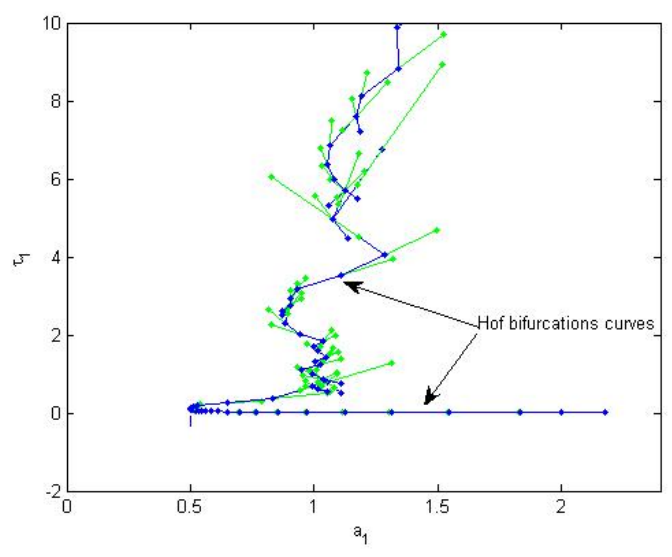

Fig. 8. Global Hopf bifurcations branches as we vary $\tau_{1}$ and $a_{1}$ (same as varying $\mathrm{R}$ ).
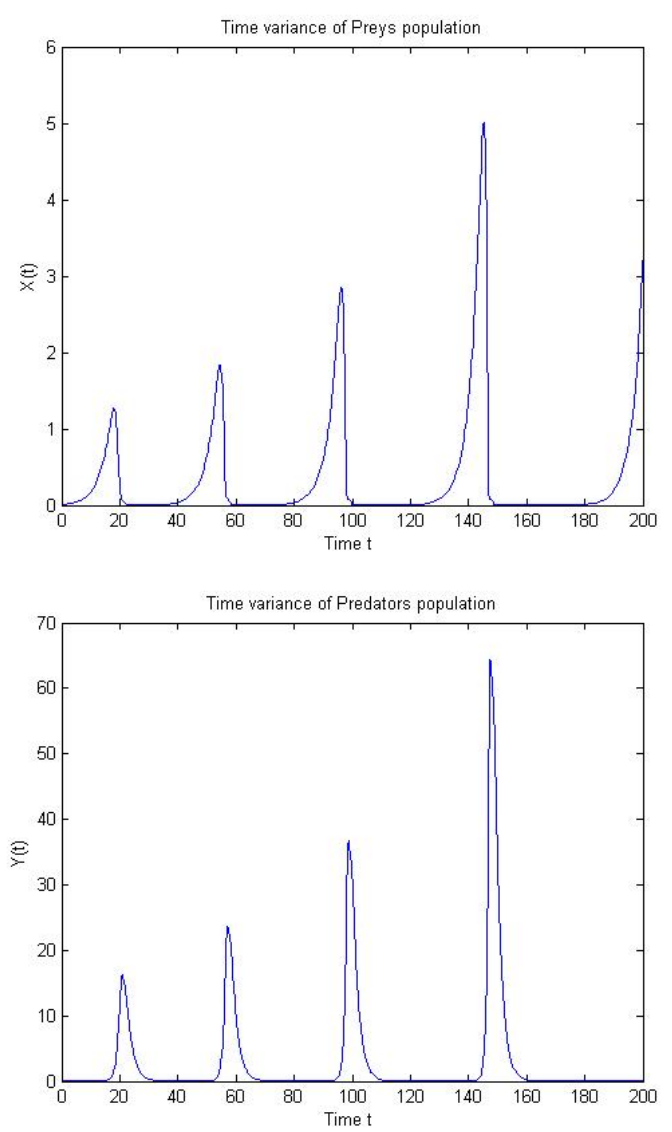

Fig. 9. The positive equilibrium is unstable for $\tau_{1}=2$ and $\tau_{2}=0.5$. 
I. Diakite et al., Effects of Discrete Time Delays and Parameters ...

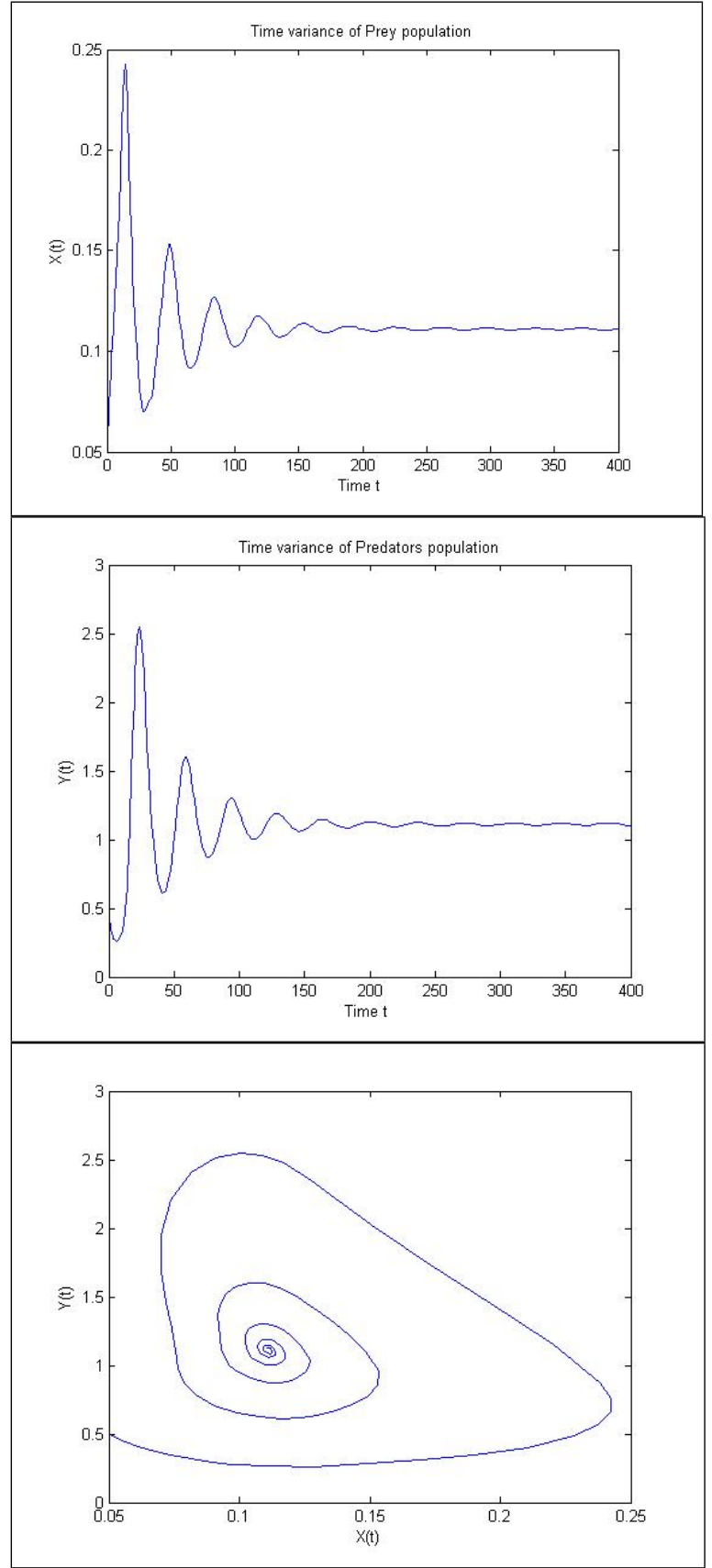

Fig. 10. The positive equilibrium is stable for $\tau_{1}=7$ and $\tau_{2}=1.2$.The system exhibits a spiral in toward the equilibrium $\left(x_{1}^{*}, y_{1}^{*}\right)=(0.111,1.111)$.

For $\tau_{1}=7$ and $\tau_{2}=1.2$ the equilibrium becomes stable as shown in Figure 10.
For the case of two non-zero delays, we use Matlab to compute numerical simulations illustrating the effects of the two delays. The analysis is summarized in Table III

TABLE III

STABILITY REGIONS IN CASE OF TWO NON-ZERO DELAYS

\begin{tabular}{|c|c|c|c|}
\hline Unstable & Stable & Stable & Unstable \\
\hline $0 \leq \tau_{1}<\tau_{1 c}^{\prime}$ & $\tau_{1}>\tau_{1 c}^{\prime}$ & $0 \leq \tau_{1}<\tau_{1 c}^{\prime}$ & $\tau_{1}>\tau_{1 c}^{\prime}$ \\
\hline $\begin{array}{l}0 \leq \tau_{2}<\tau_{2 c} \\
\text { see Fig } 4 \text { and Fiq } 9\end{array}$ & $\begin{array}{l}0 \leq \tau_{2}<\tau_{2 c} \\
\text { see Fig } 6 \text { and Fig } 10\end{array}$ & $\begin{array}{l}\tau_{2}>\tau_{2 c} \\
\quad \text { see Fis } 11\end{array}$ & $\begin{array}{l}\tau_{2}>\tau_{2 c} \\
\text { see Fif } 12\end{array}$ \\
\hline
\end{tabular}
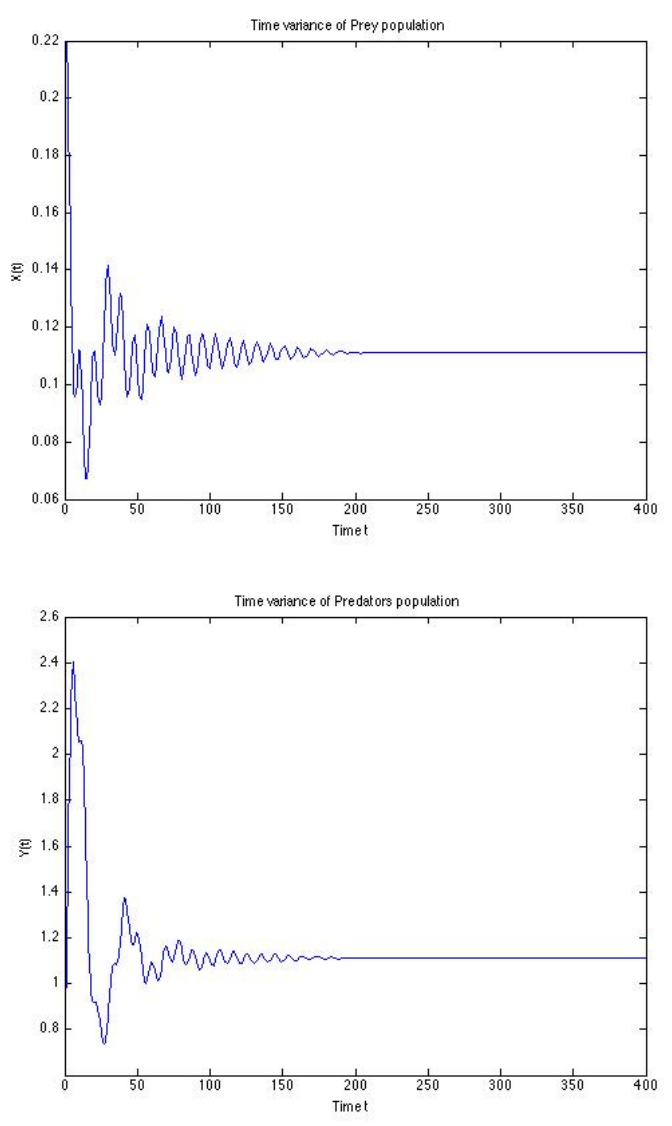

Fig. 11. The positive equilibrium is stable for $\tau_{1}=0.7$ and $\tau_{2}=8$. 


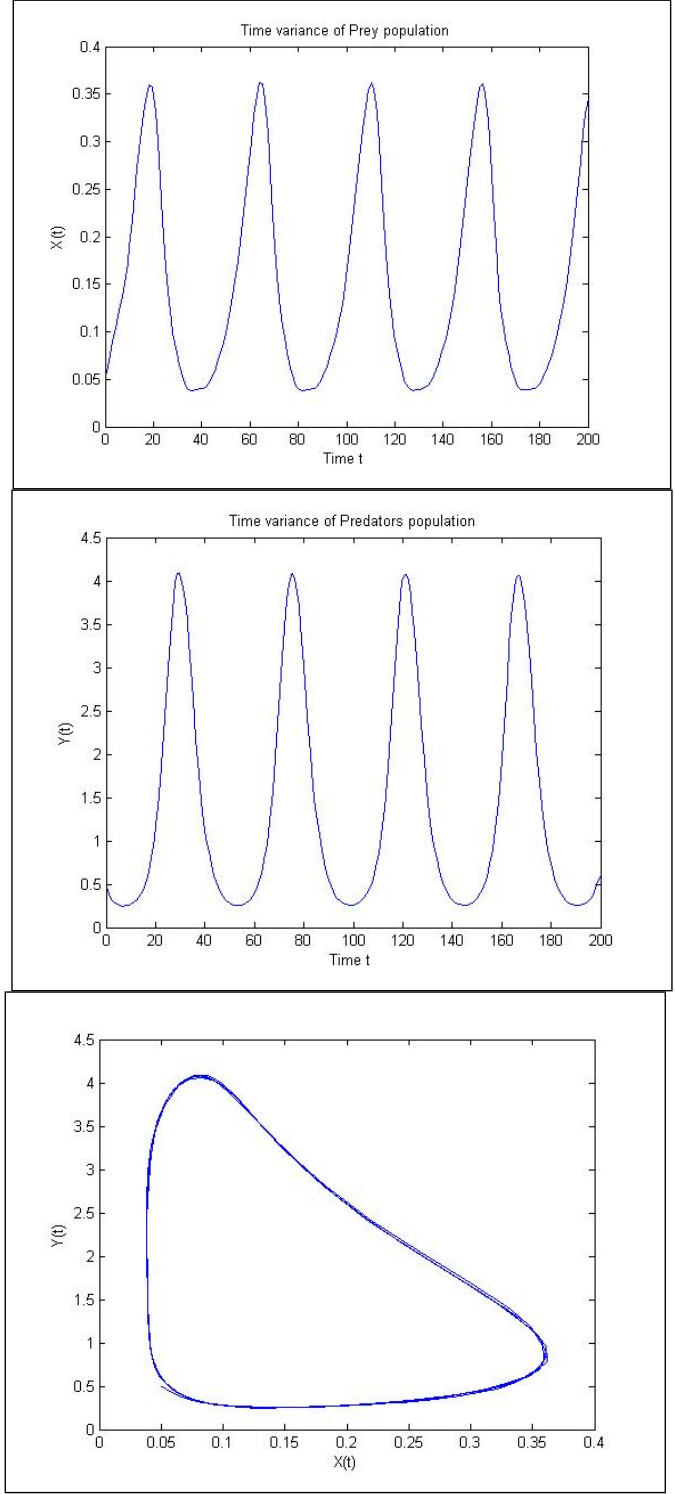

Fig. 12. The positive equilibrium is unstable for $\tau_{1}=7$ and $\tau_{2}=3.1$. The system exhibits an unstable periodic solutions.
Published papers have shown that the incorporation of discrete time delays can highly impact the dynamics of the system, since they can cause stability switches of a steady state point, and can also cause the system to go through a Hopf bifurcation near that steady state point (Culshaw[6], Bellen $[3], \ldots)$. The highlight of this paper is on how a local bifurcation parameter of the system may modify the stability changes caused by the delay(s).To understand the effects of discrete time delays and parameter variations on certain biological system models, we carried out a bifurcation analysis of a system of delay differential equations in detail for $n=1$ with specific examples, gave the procedure for higher $\mathrm{n}$, and did a concrete example for $n=2$. We investigated the stability of the steady states as both bifurcation parameters, the discrete time delay $\tau$ and a local bifurcation parameter $\mu$, cross critical values. Our analysis shows that while both parameters can destabilize the steady state, the discrete time delay can cause stability switches of the steady state only upon certain values of $\mu$. The local bifurcation parameter effects on the stability of the steady state do not depend on the value of $\tau$. We also showed that both parameters act differently in term of bifurcation. While the discrete time delay may only introduce a Hopf bifurcation, the parameter $\mu$ can introduce other type of bifurcations.

For $\tau_{1}=7$ and $\tau_{2}=3.1$ the equilibrium becomes unstable again as shown in Figure 12 .

\section{CONCLUSIONS AND Discussion}

It is well known that changes in the parameters play a crucial role in understanding dynamical systems. There is a need to incorporate discrete time delays in dynamical systems (Biological systems, physical systems,...) as has been shown and studied by many researchers (Perelson[1],Bellen[3],..). 


\section{APPEndix A}

Theorem 1: Consider the transcendental equation

$$
\lambda^{n}+\sum_{i=1}^{n} a_{n-i} \lambda^{n-i}+\sum_{i=1}^{n} b_{n-i} e^{-\lambda \tau} \lambda^{n-i}=0,
$$

if there exists a $\tau_{c}>0$ such that $\lambda\left(\tau_{c}\right)$ is a purely imaginary eigenvalue of (34), then for $\tau>\tau_{c}$ the transcendental equation (34) has at least one eigenvalue with a strictly positive real part.

Before we prove the above theorem, let just first consider a much simpler case. Consider the analytic function

$$
h(\lambda, a)=\lambda+e^{-\lambda \tau}+a,
$$

with $\tau \geq 0$, and $a \in \mathbb{R}$.

Then $h(\lambda, 0)=0$ if and only if

$$
\lambda=-e^{-\lambda \tau} .
$$

Equation (35) has purely imaginary roots if and only if $\tau=\tau_{c}=2 j \pi+\frac{\pi}{2}, j=0,1,2, \ldots$.

The proof of the following lemma can be found in Cooke and Van den Driessche [20]; see also Bellman and Cooke [4].

Lemma 2: If $\tau \in\left[0, \frac{\pi}{2}\right)$, then all roots of equation (35) have strictly negative real parts. If $\tau \in\left(2 j \pi+\frac{\pi}{2},(2 j+1) \pi+\frac{\pi}{2}\right]$, then equation (35) has exactly $2 j+1$ roots with strictly positive real parts.

We have $h(\lambda, a)$ is an analystic function in $\lambda$, $a$. When $\tau \neq 2 j \pi+\frac{\pi}{2}$, the function $h(\lambda, 0)$ has no zeros on the boundary of $\Omega$, where $\Omega=$ $\{\lambda,|\operatorname{Re}(\lambda) \geq 0,| \lambda \mid \leq \rho\}$. Thus, Rouche's theorem (Dieudonne[11], Theorem 9.17.4) implies that there exists a $\delta>0$ such that :

(1) for any $a<\delta, h(\lambda, a)$ has no zero on the boundary of $\Omega$

(2) for any $a<\delta, h(\lambda, a)$ and $h(\lambda, 0)$ have the same sum of the orders of zeros belonging to $\Omega$.

It follows from lemma 2 that when $\tau>\frac{\pi}{2}$, the sum of the orders of the zeros of $h(\lambda, 0)$ belonging to $\Omega$ is at least 1 . Thus when $\tau>\frac{\pi}{2}, \tau \neq 2 j \pi+\frac{\pi}{2}$, and $a<\delta$ then $h(\lambda, a)$ has at least a root with strictly positive real part.

Now we can prove the more general form which is theorem 1

Proof : Consider the analytic function in $\lambda, A$

$$
h(\lambda, A)=\lambda^{n}+\sum_{i=1}^{n} a_{n-i} \lambda^{n-i}+\sum_{i=1}^{n} b_{n-i} e^{-\lambda \tau} \lambda^{n-i},
$$

where $\lambda \in \mathbb{C}$, and

$$
A=\left(a_{n-1}, \ldots, a_{1}, a_{0}, b_{n-1}, \ldots, b_{1}\right) \in \mathbb{R}^{n \times(n-1)} .
$$

Then

$$
h\left(\lambda, A_{0}\right)=\lambda^{n}+b_{0} e^{-\lambda \tau}
$$

where $A_{0}=(0, \ldots, 0)$ is the null vector. $h\left(\lambda, A_{0}\right)$ has purely imaginary roots if and only if

$$
\begin{gathered}
\tau=\tau_{c}^{j}=\frac{2 j \pi}{b_{0}^{1 / n}} \\
j=1,2, \ldots \text { when } n \text { is even, }
\end{gathered}
$$

or

$$
\begin{gathered}
\tau=\tau_{c}^{j}=\frac{(4 j+1) \pi}{2 b_{0}^{1 / n}} \\
j=0,1,2, \ldots \text { when } n \text { is odd }
\end{gathered}
$$

and here we assume that $b_{0}>0$, otherwise we multiple by a - sign. When $\tau \neq \tau_{c}^{j}$ the function $h\left(\lambda, A_{0}\right)$ has no zero on the boundary of $\Omega$, where $\Omega=\{\lambda,|\operatorname{Re}(\lambda) \geq 0,| \lambda \mid \leq \rho\}$. Thus, Rouche's theorem implies that there exists a $\delta>0$ such that :

(1) when $\|A\|_{\infty}<\delta, h(\lambda, A)$ has no zero on the boundary of $\Omega$

(2) when $\|A\|_{\infty}<\delta, h(\lambda, A)$ and $h\left(\lambda, A_{0}\right)$ have the same sum of the orders of zeros belonging to $\Omega$.

It follows from lemma 2 that when $\tau>\tau_{c}=$ $\frac{2 \pi}{b_{0}^{1 / n}}$ and $\tau \neq \frac{2 j \pi}{b_{0}^{1 / n}}$ (or $\tau>\tau_{c}=\frac{1 \pi}{2 b_{0}^{1 / n}}$ and $\tau \neq$ $\frac{(4 j+1) \pi}{\left.2 b_{0}^{1 / n}\right)}$, the sum of the orders of the zeros of $h\left(\lambda, A_{0}\right)$ belonging to $\Omega$ is at least 1 . Thus when $\tau>\tau_{c}, \tau \neq \tau_{c}^{j}$ and $\|A\|_{\infty}<\delta$ then $h(\lambda, A)$ has at least a root with strictly positive real part. 


\section{APPENDIX B}

\section{A. Proof of Proposition 1}

The characteristic equation of the one dimensional DDE is given by (3) and because the steady state is assumed to be stable at $\tau=0$ then

$$
\alpha(0)=\left.\frac{d f_{1}}{d X}\right|_{\left(X^{*}, \mu^{*}\right)}+\left.\frac{d f_{2}}{d X}\right|_{\left(X^{*}, \mu^{*}\right)}<0 .
$$

If (i) holds then equation (37) implies $\left.\left|\frac{d f_{2}}{d X}\right|_{\left(X^{*}, \mu^{*}\right)}|<| \frac{d f_{1}}{d X}\right|_{\left(X^{*}, \mu^{*}\right)} \mid$

therefore equation (9) has no positive root meaning the steady state remains stable for all $\tau \geq 0$.

If (ii) holds then equation 37 implies $\left.\left|\frac{d f_{2}}{d X}\right|_{\left(X^{*}, \mu^{*}\right)}|>| \frac{d f_{1}}{d X}\right|_{\left(X^{*}, \mu^{*}\right)} \mid$

therefore equation (9) has a positive root then there exists a $\tau_{c}>0$ such that $\alpha(\tau)>0$ whenever $\tau>\tau_{c}$.

If (iii)(a) holds then again equation (9) has no solution therefore $\alpha(\tau)<0$ for all $\tau \geq 0$ meaning the steady state remains stable.

If (iii)(b) holds then equation (9) has a positive root then there exists a $\tau_{c}>0$ such that $\alpha(\tau)>0$ whenever $\tau>\tau_{c}$.

\section{B. Proof of Proposition 2}

If conditions of proposition 1(iii)(a) hold then there is nothing to prove. Assume that conditions of proposition 1(iii)(b) hold then equation (9) has a positive solution, therefore the delay can affect the stability of the equilibrium point. But if for some $\mu^{*}$ in $D_{\mu}$ we have the extra condition

$$
\left.\frac{d f_{1}}{d X}\right|_{\left(X^{*}, \mu^{*}\right)}=g\left(X^{*}\right) \mathcal{O}\left(\mu^{*}\right)
$$

and

$$
\left.\frac{d f_{2}}{d X}\right|_{\left(X^{*}, \mu^{*}\right)}=h\left(X^{*}\right) \mathcal{O}\left(\frac{1}{\mu^{*}}\right),
$$

then one can rewrite equation (9) as

$$
\omega_{c}^{2}=\left[\frac{h\left(X^{*}\right)}{\mu^{*}}\right]^{2}-\left[g\left(X^{*}\right) \mu^{*}\right]^{2} .
$$

Then there exists a critical value $\mu_{c} \in D_{\mu}$ of $\mu$ such that

$$
\frac{h\left(X^{*}\right)}{\mu^{*}} \approx 0 \quad \text { as } \quad \mu^{*} \rightarrow \mu_{c} .
$$

Therefore equation (9) becomes

$$
\omega_{c}^{2}=-\left[g\left(X^{*}\right) \mu_{c}\right]^{2}<0,
$$

which has no real positive root $\omega_{c}$, therefore $\alpha(\tau)<0$ for all $\tau \geq 0$. This implies the delay does not have any effect on the stability of the equilibrium point when $\mu^{*}>\mu_{c}$.

\section{Proof of Proposition 5}

The Jacobian matrix of the system $(24,25)$ is given by :

$$
J=\left[\begin{array}{cc}
r_{1} e^{-\lambda \tau_{1}}-\frac{a_{1} y^{*}}{\left(x^{*}+1\right)^{2}} & -\frac{a_{1} x^{*}}{x^{*}+1} \\
\frac{a_{2} y^{*}}{\left(x^{*}+1\right)^{2}} e^{-\lambda \tau_{2}} & -r_{2}+\frac{a_{2} x^{*}}{x^{*}+1} e^{-\lambda \tau_{2}}
\end{array}\right]
$$

Evaluating at $\left(x^{*}, y^{*}\right)=(0,0)$, the characteristic equation is given as

$$
\left(\lambda-r_{1} e^{-\lambda \tau_{1}}\right)\left(\lambda+r_{2}\right)=0 .
$$

We note that the stability of $\left(x^{*}, y^{*}\right)=(0,0)$ depends only on $\tau_{1}$.

- If $\tau_{1}=0$ then the eigenvalues are : $\lambda=r_{1}>0$ and $\lambda=-r_{2}<0$. Therefore the $(0,0)$ is unstable.

- If $\tau_{1}>0$, we have $\lambda=r_{1} e^{-\lambda \tau_{1}}$, let $\lambda(\tau)=$ $\alpha(\tau)+i \omega(\tau)$ then we have $\lambda=r_{1} e^{-\alpha \tau_{1}}\left(\cos \omega \tau_{1}-i \sin \omega \tau_{1}\right)$.

One can choose $\omega_{c} \tau_{1 c}=\frac{\pi(2 n+1)}{2}(\mathrm{n}=0,1,2, \ldots)$ or $\tau_{1 c}=\frac{\pi(2 n+1)}{2 \omega_{c}}$ such that the real part of $\lambda(\tau)=\alpha(\tau)+i \omega(\tau)$ at $\tau_{1 c}$ is zero $\left(\alpha\left(\tau_{1 c}\right)=\right.$ $0)$ and the imaginary part $\omega\left(\tau_{1 c}\right)=\omega_{c}$ is a solution of the characteristic equation.

Then by the continuity of $\alpha$ we have :

- $\alpha(\tau)>0$ for $\tau_{1}<\tau_{1 c}$,

- $\alpha(\tau)<0$ for $\tau_{1}>\tau_{1 c}$.

\section{Proof of Proposition 6}

The characteristic equation of the system evaluating at $\left(x_{1}^{*}, y_{1}^{*}\right)$ is given by

$$
\begin{array}{r}
\lambda^{2}+\left(b-r_{1} e^{-\lambda \tau_{1}}-r_{2} e^{-\lambda \tau_{2}}\right) \lambda+\left(f-f_{1} e^{-\lambda \tau_{1}}\right)+ \\
\left(a_{1}-1\right) f e^{-\lambda \tau_{2}}+f_{1} e^{-\lambda\left(\tau_{1}+\tau_{2}\right)}=0,
\end{array}
$$


where

$$
b=r_{2}+\frac{r_{1}}{x_{1}^{*}+1}, \quad f=\frac{r_{1} r_{2}}{x_{1}^{*}+1}, \quad f_{1}=r_{1} r_{2} .
$$

- If $\tau_{1}=\tau_{2}=0$ we have:

$$
\lambda^{2}-\left(b-r_{1}-r_{2}\right) \lambda+a_{1} f=0
$$

with

$$
b-r_{1}-r_{2}=-\frac{r_{1}}{R}<0, \quad \text { and } \quad a_{1} f>0 .
$$

Then the characteristic equation has at least a positive eigenvalue

(if the eigenvalues are real) $\lambda=\frac{r_{1}}{2 R}+\frac{\sqrt{\delta}}{2}$ where

$$
\delta=\left(b-r_{1}-d\right)^{2}-4 a_{1} f,
$$

or, all its eigenvalues (if complex) have a positive real part $\left(\frac{r_{1}}{2 R}\right)$. Therefore the steady state $\left(x_{1}^{*}, y_{1}^{*}\right)$ is unstable.

- If $\tau_{1}>0$ and $\tau_{2}=0$ Then the characteristic equation becomes:

$$
\lambda^{2}+\left(b-r_{2}\right) \lambda-r_{1} e^{-\lambda \tau_{1}} \lambda+a_{1} f=0 .
$$

Since we know that the steady state is unstable when $\tau_{1}=\tau_{2}=0$, the question becomes: does there exist a $\tau_{1 c}^{\prime}$ such that the steady state stabilizes as $\tau_{1}$ crosses $\tau_{1 c}^{\prime}$ ? In other words if $\lambda\left(\tau_{1}\right)=\alpha\left(\tau_{1}\right)+i \omega\left(\tau_{1}\right)$, does there exist $\tau_{1 c}^{\prime}$ such that $\alpha\left(\tau_{1 c}^{\prime}\right)=0$ and $\omega\left(\tau_{1 c}^{\prime}\right)=\omega_{c}^{\prime}$ which satisfies

$$
\begin{array}{r}
-\omega_{c}^{\prime 2}+i\left(b-r_{2}\right) \omega_{c}^{\prime}- \\
i r_{1} \omega_{c}^{\prime}\left(\cos \omega_{c}^{\prime} \tau_{1 c}^{\prime}-i \sin \omega_{c}^{\prime} \tau_{1 c}^{\prime}\right)+a_{1} f=0 .
\end{array}
$$

Setting the real and imaginary parts equal zero, we obtain:

$$
\begin{gathered}
-\omega_{c}^{\prime 2}+a_{1} f=r_{1} \omega_{c}^{\prime} \sin \omega_{c}^{\prime} \tau_{1 c}^{\prime} \\
(b-d) \omega_{c}^{\prime}=r_{1} \omega_{c}^{\prime} \cos \omega_{c}^{\prime} \tau_{1 c}^{\prime} .
\end{gathered}
$$

Adding the square of both equations, we obtain:

$\omega_{c}^{\prime 4}+\left[\left(b-r_{2}\right)^{2}-r_{1}^{2}-2 a_{1} f\right] \omega_{c}^{\prime 2}+a_{1}^{2} f^{2}=0$

Such $\tau_{1 c}^{\prime}$ exists if and only if the above equation has at least a positive root $\omega_{c}^{\prime}$. Let $M=\omega_{c}^{\prime 2}$, then we have the quadratic equation:

$M^{2}+\left[\left(b-r_{2}\right)^{2}-r_{1}^{2}-2 a_{1} f\right] M+a_{1}^{2} f^{2}=0$

which has at least a positive root if:

$C(0): \quad\left[\left(b-r_{2}\right)^{2}-r_{1}^{2}-2 a_{1} f\right]<0$, and $\Delta=\left[\left(b-r_{2}\right)^{2}-r_{1}^{2}-2 a_{1} f\right]^{2}-4 a_{1}^{2} f^{2} \geq$ 0 , consequently, equation (42) has at least a positive root $\omega_{c}^{\prime}$. Which implies there exist a $\tau_{1 c}^{\prime}>0$ such that the steady state changes stability as $\tau_{1}$ crosses $\tau_{1 c}^{\prime}$ for $\tau_{2}=0$. In fact $\tau_{1 c}^{\prime}$ is the smallest of :

$\tau_{1 c}^{\prime j}=\frac{1}{\omega_{c}^{\prime}} \arccos \frac{b-r_{2}}{r_{1}}+\frac{2 \pi j}{\omega_{c}^{\prime}}, \quad j=1,2, \ldots \square$

\section{REFERENCES}

[1] A. S. Perelson, D. E. Kirschner, R. DE Boer, Dynamics of HIV infection of CD4+ T cells, Math Biosci. 114 (1993) 81-125

[2] L. Allen, An Introduction to Mathematical Biology, Pearson-Prentice Hall, Upper Saddle River, NJ, 2007.

[3] A. Bellen, M. Zennaro, Numerical Methods for Delay Differential equations, Oxford University Press, NY 2005.

[4] R. Bellman, K. L. Cooke, Differential-Difference Equations, Academic Press, New York, 1963.

[5] Driver, Rodney D. Ordinary and Delay Differential Equations, New York: Springer Verlag (1977). ISBN 0387-90231-7.

[6] R. V. Culshaw, S. Ruan, A delay-differential equation model of HIV infection of $\mathrm{CD}^{+}{ }^{+}$T-cells, Mathematical Biosciences 165 (2000) 27-39.

[7] S. Gakkhar, A. Singh, Complex dynamics in a prey predator system with multiple delays, Commun Nonlinear SCi Numer Simulat. 17 (2012) 914-929.

[8] K. Gopalsamy Stability and Oscillations in Delay Differential Equations of Population Dynamics, Kluwer Academics Publishers, 1992.

[9] S. Ruan, On Nonlinear Dynamics of Predator-Prey Models with discrete Delay, Math. Model. Nat. Phenom. 4 (2009) 140-188. 
I. Diakite et al., Effects of Discrete Time Delays and Parameters ...

[10] M. Y. Li, H. Shu, Joint effects of mitosis and intracellular delay on viral dynamics:two-parameter bifurcation analysis, Math. Biol. 64 (2011) 1005-20.

[11] J. Dieudonne, Foundations of Modern Analysis, Academic Press, New York. 1960

[12] J. Guckenheimer, P. Holmes, Nonlinear Oscillations, Dynamical Systems, and Bifurcations of Vector Fields, Springer-Verlag, New York, 1997.

[13] J. K. Hale, S. M. V. Lunel, Introduction to Functional Differential Equations, Springer Verlag, New York, 1993.

[14] Y. S. Chin, Unconditional stability of systems with timelags., Acta Math. Sinica, 1 (1960) 138-155.

[15] R. Datko, A procedure for determination of the exponential stability of certain differential difference equations. Quart. Appl. Math. 36 (1978) 279-292.

[16] J. E. Ameh and R. Ouifki, The Basic Reproductive Num- ber: Bifurcation and Stability, Thesis, African Institute for Mathematical Sciences. 2009.

[17] K. Engelborghs, T. Luzyanina, G. Samaey, DDEBIFTOOL v. 2.00: a Matlab package for bifurcation analysis of delay differential equations, Report TW 330, 2001.

[18] L. F. Shampine, I. Gladwell, S. Thompson,Solving ODEs with MATLAB, Cambridge University Press, Cambridge 2003.

[19] L. F. Shampine, S. Thompson, Solving DDEs in MAT$L A B$, Applied Numerical Mathematics 37 (2001) 441458.

[20] K.L. Cooke, P.vanden Driessche, and X. Zou, Interaction of maturation delay and nonlinear birth in population and epidemic models, J. Math. Biol, 39 (1999) 332-352. 